\title{
Breath-holding as a novel approach to risk stratification in COVID-19
}

\author{
Ludovico Messineo ${ }^{1,2^{*}+}$ (D), Elisa Perger ${ }^{3,4 \dagger}$, Luciano Corda 5,6 , Simon A. Joosten ${ }^{7,8,9}$, Francesco Fanfulla ${ }^{10}$, \\ Leonardo Pedroni ${ }^{5}$, Philip I. Terrill ${ }^{11}$, Carolina Lombardi ${ }^{3,4}$, Andrew Wellman ${ }^{1}$, Garun S. Hamilton ${ }^{7,8,9}$, \\ Atul Malhotra ${ }^{12}$, Guido Vailati ${ }^{5}$, Gianfranco Parati ${ }^{3,4}$ and Scott A. Sands ${ }^{1,13}$
}

\begin{abstract}
Background: Despite considerable progress, it remains unclear why some patients admitted for COVID-19 develop adverse outcomes while others recover spontaneously. Clues may lie with the predisposition to hypoxemia or unexpected absence of dyspnea ('silent hypoxemia') in some patients who later develop respiratory failure. Using a recently-validated breath-holding technique, we sought to test the hypothesis that gas exchange and ventilatory control deficits observed at admission are associated with subsequent adverse COVID-19 outcomes (composite primary outcome: non-invasive ventilatory support, intensive care admission, or death).
\end{abstract}

Methods: Patients with COVID-19 ( $N=50)$ performed breath-holds to obtain measurements reflecting the predisposition to oxygen desaturation (mean desaturation after 20-s) and reduced chemosensitivity to hypoxic-hypercapnia (including maximal breath-hold duration). Associations with the primary composite outcome were modeled adjusting for baseline oxygen saturation, obesity, sex, age, and prior cardiovascular disease. Healthy controls $(N=23)$ provided a normative comparison.

Results: The adverse composite outcome (observed in $N=11 / 50$ ) was associated with breath-holding measures at admission (likelihood ratio test, $p=0.020$ ); specifically, greater mean desaturation (12-fold greater odds of adverse composite outcome with $4 \%$ compared with $2 \%$ desaturation, $p=0.002$ ) and greater maximal breath-holding duration (2.7-fold greater odds per 10-s increase, $p=0.036$ ). COVID-19 patients who did not develop the adverse composite outcome had similar mean desaturation to healthy controls.

Conclusions: Breath-holding offers a novel method to identify patients with high risk of respiratory failure in COVID19. Greater breath-hold induced desaturation (gas exchange deficit) and greater breath-holding tolerance (ventilatory control deficit) may be independent harbingers of progression to severe disease.

Keywords: Desaturation, Hypoxia, Chemosensitivity, Dyspnea, Prognosis

*Correspondence: ludovico.messineo@yahoo.it

†Ludovico Messineo and Elisa Perger have equaly contributed to this work

${ }^{1}$ Division of Sleep and Circadian Disorders, Departments of Medicine and Neurology, Brigham \& Women's Hospital \& Harvard Medical School, Boston, MA, USA

Full list of author information is available at the end of the article

\section{Background}

COVID-19 outcomes are notoriously unpredictable [1]: roughly $5 \%$ of patients exhibit severe and progressive pneumonia that requires intensive care in the form of mechanical ventilatory support [2]. Favorable outcomes of serious cases rely on judicious administration of ventilatory support for those likely to benefit most [3], but such resources have become uniquely limited during peaks in the pandemic. While known risk factors original author(s) and the source, provide a link to the Creative Commons licence, and indicate if changes were made. The images or other third party material in this article are included in the article's Creative Commons licence, unless indicated otherwise in a credit line to the material. If material is not included in the article's Creative Commons licence and your intended use is not permitted by statutory regulation or exceeds the permitted use, you will need to obtain permission directly from the copyright holder. To view a copy of this licence, visit http://creativecommons.org/licenses/by/4.0/. The Creative Commons Public Domain Dedication waiver (http://creativeco mmons.org/publicdomain/zero/1.0/) applies to the data made available in this article, unless otherwise stated in a credit line to the data. 
for adverse outcomes include older age [2, 4-7], lower baseline pulse oxygen saturation $\left(\mathrm{SpO}_{2}\right)[5,7,8]$, obesity [4], cardiovascular comorbidities [4-6], and inflammatory biomarkers (e.g. C-reactive protein [6]), considerable unexplained heterogeneity remains [5, 9]. A deeper understanding of why some patients deteriorate after admission while others recover with minimal intervention is needed. Such knowledge could help facilitate earlier administration and prioritization of advanced emerging medical interventions.

One of the most consistent risk factors for adverse outcomes of COVID-19 is lower baseline $\mathrm{SpO}_{2}[5,7,8]$, a reflection of disease-related gas exchange deficits (e.g. ventilation/perfusion $[\mathrm{V} / \mathrm{Q}]$ heterogeneity). In addition to baseline $\mathrm{SpO}_{2}$, reports from Italy early in the pandemic described successful triage of patients using exertional desaturation (cardiometabolic challenge) [10-12] as a means to reveal gas exchange abnormalities. However, the role of exertional desaturation as a risk factor independent of baseline $\mathrm{SpO}_{2}$ remains unproven [12, 13]. Ground glass opacities and consolidation seen in computed tomography prior to respiratory failure $[5,14,15]$ suggest that gas exchange deficits are a likely risk factor. Yet, to date, the propensity for additional rapid desaturation with a ventilatory challenge (breath-holding) has not been examined as a risk factor. In principle, breathholding is expected to yield rapid desaturation in those with early gas exchange deficits (V/Q heterogeneity and reduced functional lung gas volumes [16-18]) beyond baseline $\mathrm{SpO}_{2}$. Moreover, in the context of anecdotal reports of "silent hypoxemia" (disproportionate tolerance of hypoxemia) as a characteristic of COVID-19 $[19,20]$, we and others $[21,22]$ considered that blunted ventilatory control (reduced chemosensitivity) may be an adverse neurophysiological consequence of infection, and could feasibly predispose to respiratory failure. By contrast, others have suggested that a robust ventilatory responses may promote patient self-inflicted lung injury (P-SILI) [23, 24], although this notion remains contentious [25]. There is a lack of available physiological data on the risks of respiratory failure associated with blunted $v$. robust ventilatory control in patients with COVID-19.

Accordingly, in a prospective, multi-center, observational study, we aimed to determine whether gas exchange and ventilatory control deficits in patients admitted for COVID-19 are associated with adverse outcomes of the disease (primary composite outcome of non-invasive pressure support, intensive care admission, or death). We used a simple, non-invasive, recently-validated breath-holding technique [26] to test the hypothesis that adverse outcomes are independently associated with (1) greater mean desaturation during a fixed-duration (20-s) breath-hold, and (2) reduced chemosensitivity based on greater maximal breath-hold duration [26-28]. The magnitude of the spontaneous ventilatory response following 20-s breath-holds (lower in those with reduced chemoreflex sensitivity) was also evaluated as a risk factor [26].

\section{Methods}

\section{Participant recruitment}

Fifty-seven hospitalized patients aged 18-90 year were enrolled after clinical diagnosis of COVID-19 in three different centers in northern Italy (Brescia, Milan, Pavia). Diagnosis was confirmed with a positive nasal or pharyngeal swab or with clear clinical evidence (i.e. typical signs at laboratory blood tests and computed tomography and/or chest ultrasound) when the swab result was yet not available (swab positivity was confirmed in all patients eventually). Exclusion criteria were: more-thanmoderate dyspnea (Borg $\geq 4$ ), hemodynamic instability, Brescia-COVID respiratory severity scale $>1$ [29], diurnal home treatment with supplemental oxygen or ventilatory support, use of sedatives, opioids, anti-emetics or other drugs known to impact chemosensitivity, heart failure, chronic obstructive pulmonary disease, pregnancy, and inability to understand the informed consent.

Twenty-four healthy controls were contemporaneously studied in Melbourne (Monash Health), which at the time had low case rates of COVID-19. Absence of COVID-19 was assessed by medical examination. Exclusion criteria also included professional divers, singers, or trumpeters.

\section{Breath-holding procedure and analysis}

In patients, tests were performed shortly after admission, while breathing room air.

Breath-holding maneuvers were performed as described previously [26]. Briefly, while supine, participants were instructed to breathe only through the nose, hold their breath starting from residual functional capacity (FRC) and avoid deep inspiration prior to breathholds. Ambulatory equipment designed for diagnosis of sleep apnea was used: nasal flow was recorded via an uncalibrated nasal cannula, together with digital saturation recorded via a probe with signal averaging time of 3 -s or faster (8000J, Nonin, Plymouth, MN). Investigators requested $\geq 4$ reliable 20-s fixed-time breath-holds and $\geq 1$ maximal breath-hold (up to $90 \mathrm{~s}$ ). Additional details are provided in Additional file 1.

Three physiological measurements were calculated (custom MATLAB software): mean desaturation (change from baseline in $\mathrm{SpO}_{2}$ after 20-s of apnea, using ensemble averaging and delay-correction), maximal breath-hold duration (largest value observed) [26], and ventilatory response (ventilatory overshoot at the second recovery 
breath following the 20-s breath-hold; ensemble-averaged tidal volume $\times$ rate; percent of pre-breath-hold baseline [26]). Participant flow chart is illustrated in Fig. 1.

\section{Statistical analysis}

The adverse composite outcome was reached with any occurrence of non-invasive bi-level pressure support, intensive care admission, or death. Since patients with COVID-19 who met the criteria for the adverse primary outcome ultimately received ventilatory support (noninvasive or via intubation in intensive care), we refer to these individuals herein as VS+; patients discharged without experiencing the adverse primary outcome were labelled VS - (Fig. 1).

The primary hypothesis was quantified by comparing the fully adjusted model against a reference model without breath-holding measurements using a likelihood ratio test. Significance of this single test $(p<0.05)$ was taken to indicate that the 3 measurements (together) explained unique heterogeneity in the primary adverse outcome after that already explained by 5 primary model covariates (baseline $\mathrm{SpO}_{2}$, BMI, age, sex, cardiovascular disease). Subsequent analysis then examined associations with individual breath-holding measures.

Additional serial modeling analysis examined associations between the adverse composite outcome and individual breath-holding measures, with progressive adjustment for other breath-holding measures and covariates. Multivariable linear regression models also examined differences in breath-holding measures between $\mathrm{VS}+$, VS -, and controls, adjusting for covariates. Baseline $\mathrm{SpO}_{2}$ and mean desaturation were considered confounders in the analysis of associations between outcomes and breath-holding duration (i.e. greater desaturation was considered a confounding source of shorter breath-hold duration independent of chemosensitivity [30]).

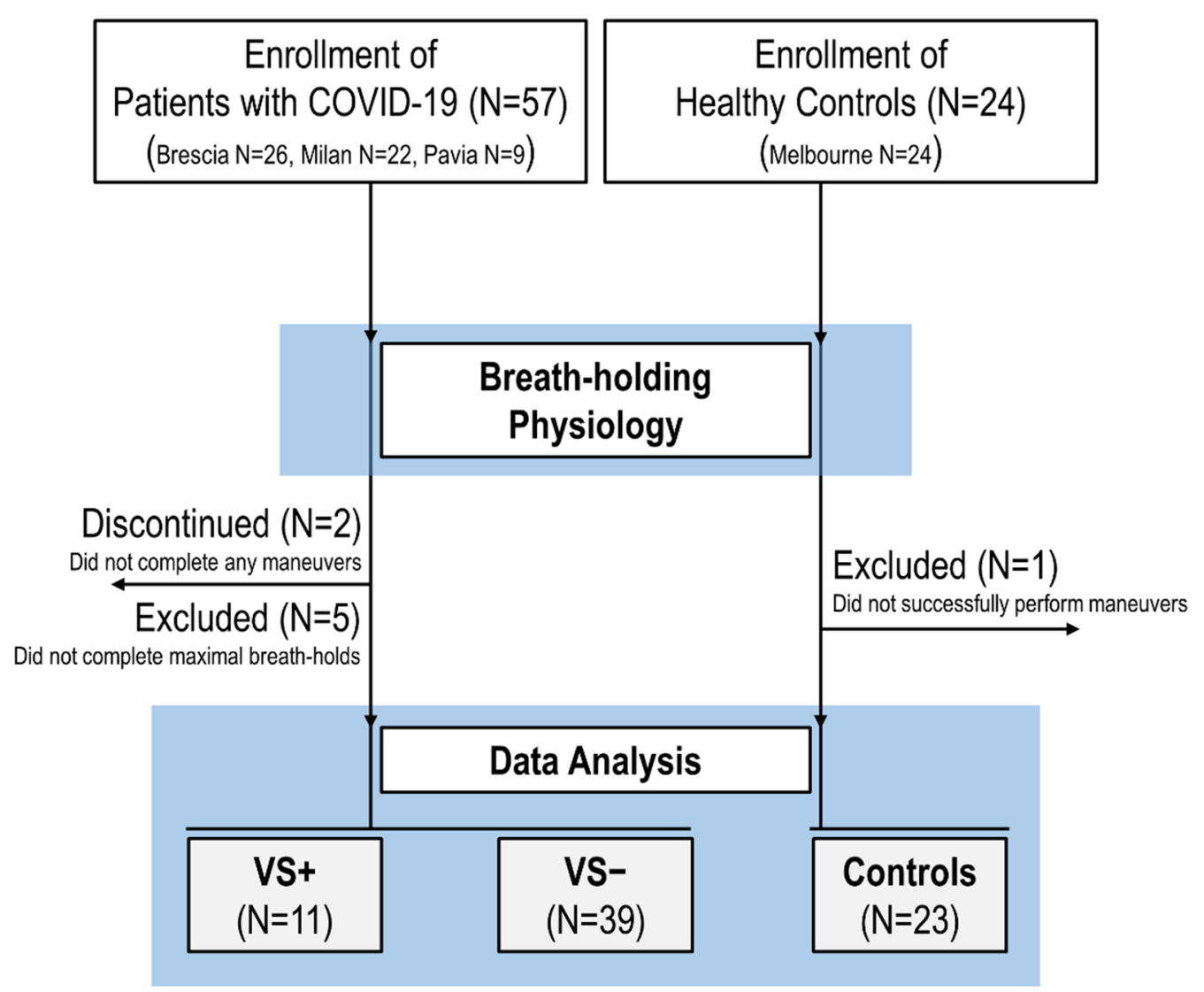

Fig. 1 Participant flow diagram. Due to discomfort related to the procedures, $N=2$ COVID-19 patients aborted the study before completing any breath-hold, $N=5$ did not complete maximal breath-holds, leaving 50/57 with available data. $N=1$ control could not complete any breath-holding. We note that (exploratory) re-inclusion of the $N=5$ patients who had available mean desaturation data (but not maximal duration variables) had no meaningful impact on the associations between mean desaturation and adverse outcomes. Patients who met the criteria for the adverse primary composite outcome are denoted "VS + " $N=4$ non-invasive bi-level pressure support, $N=7$ intensive care, $N=1$ death; "VS - "indicates patients discharged without meeting adverse primary outcome criteria 
A parsimonious model was also developed (removed covariates with high uncertainty per $p>0.2$ ) to reduce uncertainty in the remaining model coefficients.

To illustrate that the knowledge provided could potentially help with the development of a future prediction tool, we assessed the preliminary prognostic value (discriminatory capacity) of the parsimonious model, understanding the limitations of the small dataset for this purpose. Accuracy was examined with versus without the breath-holding measures (random perturbation analysis and cross-validation).

Further details, including a priori power analysis to support the sample size, are available in the Additional file 1.

\section{Role of the funding source}

This study was not directly funded.

\section{Results}

Of those enrolled, 50/57 patients with COVID-19 and 23/24 non-COVID controls completed testing and provided data for analysis (Additional file 1). Breath-holding tests were performed on the day of enrolment. Baseline characteristics of the analyzed participants are shown in Table 1. Overall, $N=11 / 50$ patients with COVID-19 met the primary composite outcome criteria (ventilatory support, intensive care, or death).

\section{Primary outcome assessment Pre-specified primary analysis}

Within COVID-19, breath-holding variables explained unique heterogeneity in the primary composite outcome adjusting for covariates (likelihood ratio 0.0073, $p=0.02$ v. model without breath-holding measures; see Table 2). Specifically: (1) the odds of the composite outcome increased 3.6 -fold per $1 \%_{\mathrm{Hb}}$ greater mean desaturation (log-odds $[\beta \pm \mathrm{SEM}]=1.27 \pm 0.59$ per $\left.\%_{\mathrm{Hb}}, p=0.002\right)$. (2) A 10-s longer maximal breath-hold duration raised odds of the primary outcome 2.7-fold ( $\log$-odds $=0.10 \pm 0.05$ per sec, $p=0.037)$. Note these odds are adjusted for baseline $\mathrm{SpO}_{2}$ and other covariates. However, the ventilatory response variable was not associated with the primary outcome. Significance was not altered by exploratory inclusion of additional available covariates to the reference model (e.g. C-reactive protein, d-dimer, hemoglobin) or omission of existing covariates (Additional file 1).

\section{Sensitivity analysis}

Greater mean desaturation raised the odds of the adverse composite outcome in simple bivariate analysis (logodds of VS+: $0.67 \pm 0.23$ per $\left.\%_{\mathrm{Hb}}, p=0.0007\right)$, when additionally adjusted for aforementioned covariates including baseline $\mathrm{SpO}_{2}(0.65 \pm 0.33, p=0.023)$, and with further adjustment for maximal breath-hold duration $(1.26 \pm 0.56, p=0.002)$. By contrast, maximal breathhold duration was only associated with the primary outcome with (but not without) the appropriate adjustment for mean desaturation (Table 2).

\section{Group differences}

In adjusted analysis, VS + patients $(N=11)$ exhibited greater mean desaturation $v . \quad \mathrm{VS}$-patients $(N=39$; difference $\left.[95 \% \mathrm{CI}] 1.6[0.3-2.8] \%_{\mathrm{Hb}}, p=0.009\right)$ and controls $\left(N=23 ; 2.3[0.8-3.9] \%_{\mathrm{Hb}}, p=0.002\right.$; Fig. $\left.2 \mathrm{~A}\right)$; differences were clear despite adjustment for the lower baseline $\mathrm{SpO}_{2}$ seen in VS+(versus VS-: 5.8[3.5-8.0] $\%_{\mathrm{Hb}}$, versus controls: $7.5[4.7-10.3] \%_{\mathrm{Hb}}, p<0.0001$, see Fig. 1B). VS - patients, but not VS + patients, had shorter adjusted maximal breath-hold duration than controls (difference $=15.9[5.6-26.1] \mathrm{s}, p=0.002$, Fig. 2C).

\section{Potential for outcome discrimination in COVID-19}

The parsimonious model (Table 2) describing risks associated with mean desaturation and maximal breath-hold duration exhibited discriminative potential (Fig. 2D-left): model accuracy was $94[88-100] \%(p<0.0001 v$. chance), which was significantly superior (random permutation analysis, $p=0.007$ ) to a reference model (covariates only; includes baseline $\mathrm{SpO}_{2}$ ) without breath-holding measurements (reference accuracy $=80[69-91] \%$, Fig. 2D-right). The breath-holding model-but not the reference model-passed cross validation analysis (accuracy $80[69-91] \%, p=0.003$; reference model accuracy $70[57-$ $83] \%, p=0.24)$.

\section{Relationship to dyspnea}

Given associations between adverse outcomes and greater breath-holding tolerance (maximal breath-hold duration) we performed additional analysis of dyspnea at admission: In fully adjusted analysis, dyspnea (Borg 1-3 $v$. Borg $=0$ ) was also associated with reduced risk of the primary outcome in COVID-19 in the current sample ([log-odds: $-2.8 \pm 1.6, p=0.038$ ). Inclusion of dyspnea in the above models did not meaningfully alter the findings reported above (see Additional file 1).

\section{Discussion}

Our prospective observational study demonstrated that adverse outcomes of COVID-19 (composite outcome of non-invasive ventilation, intensive care admission, or death) are associated with deficits in gas exchange and ventilatory control revealed using a validated breathholding technique. Specifically, we demonstrated that with increasing predisposition to oxygen desaturation during breath-holding there is higher risk of progression 
Table 1 Baseline characteristics

\begin{tabular}{|c|c|c|c|c|}
\hline \multirow[t]{2}{*}{ Characteristic } & \multicolumn{3}{|c|}{ COVID-19 patients } & \multirow{2}{*}{$\begin{array}{l}\text { Controls } \\
(N=23)\end{array}$} \\
\hline & $\begin{array}{l}\text { All } \\
(N=50)\end{array}$ & $\begin{array}{l}\text { VS }+ \\
(N=11)\end{array}$ & $\begin{array}{l}\text { VS }- \\
(N=39)\end{array}$ & \\
\hline \multicolumn{5}{|l|}{ Population factors } \\
\hline Age, years & $59.6 \pm 13.6$ & $62.7 \pm 7.5$ & $58.8 \pm 15.0$ & $45.4 \pm 10.6$ \\
\hline Female sex, $n(\%)$ & $8(16)$ & $0(0.0)$ & $8(20.5)$ & $14(60.9)$ \\
\hline Body mass index, $\mathrm{kg} / \mathrm{m}^{2}$ & $26.4 \pm 4.0$ & $28.1 \pm 4.2$ & $25.9 \pm 3.4$ & $25.2 \pm 4.7$ \\
\hline Caucasian or white race/ethnicity, $n$ (\%) & $44(88)$ & $10(90.9)$ & $34(87.2)$ & $19(82.6)$ \\
\hline \multicolumn{5}{|l|}{ History } \\
\hline History of hypertension, $n(\%)$ & $26(52)$ & $9(81.8)$ & $17(43.6)$ & $1(4.3)$ \\
\hline History of cardiovascular disease, $n(\%)$ & $4(8)$ & $1(9.1)$ & $3(7.7)$ & $0(0.0)$ \\
\hline History of diabetes, $n$ (\%) & $8(16)$ & $2(18.2)$ & $6(15.4)$ & $0(0.0)$ \\
\hline Current smoking, $n(\%)$ & $6(12)$ & $3(27.3)$ & $3(7.7)$ & $0(0.0)$ \\
\hline \multicolumn{5}{|l|}{ Current medications } \\
\hline$\beta$-blockers, $n(\%)$ & $4(8)$ & $1(9.1)$ & $3(7.7)$ & $0(0.0)$ \\
\hline ACE-inhibitors, $n(\%)$ & $9(18)$ & $4(36.4)$ & $5(12.2)$ & $0(0.0)$ \\
\hline ARB, $n(\%)$ & $4(8)$ & $2(18.2)$ & $2(5.1)$ & $0(0.0)$ \\
\hline \multicolumn{5}{|l|}{ Clinical presentation at admission } \\
\hline Baseline $\mathrm{SpO}_{2}, \%$ & $94.5 \pm 4.6$ & $89.9 \pm 6.6$ & $95.8 \pm 2.3$ & $97.6 \pm 1.0$ \\
\hline $\mathrm{PaO}_{2}$ at admission, $\mathrm{mmHg}^{*}$ & $76.2 \pm 15.3$ & $71.0 \pm 16.4$ & $77.7 \pm 14.9$ & - \\
\hline $\mathrm{PaCO}_{2}$ at admission, $\mathrm{mmHg}^{*}$ & $36.4 \pm 5.4$ & $37.0 \pm 4.6$ & $36.2 \pm 6.0$ & - \\
\hline Baseline Heart rate, beats/min & $86.8 \pm 16.8$ & $86.2 \pm 21.0$ & $86.9 \pm 13.6$ & - \\
\hline Baseline systolic blood pressure, $\mathrm{mmHg}$ & $127.3 \pm 19.1$ & $126.5 \pm 18.8$ & $127.5 \pm 19.8$ & - \\
\hline Baseline diastolic blood pressure, $\mathrm{mmHg}$ & $78.5 \pm 10.8$ & $74.4 \pm 12.5$ & $79.7 \pm 10.2$ & - \\
\hline Anosmia, $n(\%)$ & $11(22.0)$ & $1(9.1)$ & $10(25.6)$ & - \\
\hline Ageusia, $n(\%)$ & $12(24.0)$ & $2(18.2)$ & $10(25.6)$ & - \\
\hline Gastrointestinal symptoms, $n(\%)$ & $13(26.0)$ & $2(18.2)$ & $11(28.2)$ & - \\
\hline Dyspnea presence, $n(\%)$ & $13(26.0)$ & $3(27.3)$ & $10(25.6)$ & - \\
\hline \multicolumn{5}{|l|}{ Laboratory tests } \\
\hline C-reactive protein, $\mathrm{mg} / \mathrm{L}$ & $20.2 \pm 41.9$ & $14.6 \pm 23.7$ & $21.8 \pm 15.0$ & - \\
\hline D-dimer, $\mu \mathrm{g} / \mathrm{L}$ & $0.8 \pm 0.8$ & $1.1 \pm 0.8$ & $0.7 \pm 0.9$ & - \\
\hline \multicolumn{5}{|l|}{ Breath-holding measurements } \\
\hline Mean desaturation, $\%$ & $2.34 \pm 1.78$ & $4.42 \pm 2.37$ & $2.21 \pm 1.43$ & $1.57 \pm 1.24$ \\
\hline Maximal breath-hold duration ${ }^{* *}, \mathrm{~s}$ & $46.8 \pm 16.9$ & $51.8 \pm 12.7$ & $41.8 \pm 14.2$ & $53.0 \pm 20.3$ \\
\hline Recovery breath, $\%_{\text {baseline }}$ & $188.9 \pm 61.3$ & $184.6 \pm 81.8$ & $195.0 \pm 62.1$ & $180.5 \pm 49.4$ \\
\hline \multicolumn{5}{|l|}{ Outcomes } \\
\hline Discharge:oxygen:ventilatory support, $n$ & 14:36:11 & $0: 11: 11$ & 14:25:0 & - \\
\hline Duration of hospitalization, days & $29.8 \pm 17.2$ & $47.4 \pm 12.5$ & $24.8 \pm 15.3$ & - \\
\hline
\end{tabular}

Data are expressed as mean \pm SD or as $N(\%)$. Patients who met the criteria for the adverse primary composite outcome are denoted "VS + " ( $N=4$ non-invasive bi-level pressure support, $N=7$ intensive care, $N=1$ death). In all patients, diagnosis was confirmed with a positive nasal or pharyngeal swab. All patients admitted to ICU were administered mechanical ventilatory support; the patient who died was also in intensive care on mechanical ventilatory support. "VS - " indicates patients discharged without meeting adverse primary outcome criteria. Average time to the primary outcome in VS + patients was 6 [2] days (median [IQR]). NonCaucasian/Non-White race/ethnicities were black (1 VS + and 1 VS - COVID-19 patients), Hispanic (3 VS + COVID-19 patients) and Asian (1 VS - COVID-19 patient and 4 controls). Note that participants included in the table are those who provided data for all breath-holding measures. "Discharge" indicates discharge without oxygen or interventions that met criteria for the primary outcome during the hospital stay. On average, $3.8 \pm 1.020$-s breath-holds per individual were analyzed to calculate the ventilatory response variable ( $3.6 \pm 0.9$ in controls); $1.6 \pm 0.6$ maximal breath-holds were used to determine maximal breath-hold duration ( $2.1 \pm 0.5$ in controls); $5.2 \pm 1.1$ breath-holds ( 20 -s or maximal) were used to calculate mean desaturation ( $6.3 \pm 1.1$ in controls). *Data available for 42 patients ( 9 VS + and 33 VS - ). Abbreviations: $\mathrm{SpO}_{2}$, peripheral oxyhemoglobin saturation; $\mathrm{PaO}_{2}$, partial pressure of oxygen; $\mathrm{PaCO}_{2}$, partial pressure of carbon dioxide; $\mathrm{ACE}$, angiotensin converting enzyme; ARB, angiotensin II receptor blockers. ${ }^{* *}$ Data shown are adjusted for baseline $\mathrm{SpO}_{2}$ and mean desaturation (regression)

to severe disease, independent of baseline oxygenation, and other key covariates. Greater maximal breathhold duration-adjusted for hypoxemia (i.e. baseline saturation) - was also an independent risk factor among patients with COVID-19. Our finding that blunted ventilatory control [26-28] is a deleterious physiological 
Table 2 Association between breath-holding measurements and adverse outcomes of COVID-19

\begin{tabular}{ccl}
\hline & $\begin{array}{l}\text { Primary model } \\
\boldsymbol{\beta} \pm \text { SEM } \\
(\boldsymbol{p} \text { value })\end{array}$ & $\begin{array}{l}\text { Parsimonious model } \\
\boldsymbol{\beta} \pm \text { SEM } \\
(\boldsymbol{p} \text { value })\end{array}$ \\
\hline Breath-holding measurements & & \\
Mean desaturation $(\% \mathrm{Hb})$ & $1.27 \pm 0.59$ & $1.25 \pm 0.54$ \\
& $(0.002)$ & $(0.001)$ \\
Maximal breath-hold dura- & $0.10 \pm 0.05$ & $0.10 \pm 0.05$ \\
tion (s) & $(0.037)$ & $(0.020)$ \\
Ventilatory response & $0.00 \pm 0.01$ & - \\
(\% baseline & $(0.9)$ & \\
Covariates & & \\
Body mass index (kg/m $\left.{ }^{2}\right)$ & $0.22 \pm 0.22$ & $0.27 \pm 0.19$ \\
& $(0.3)$ & $(0.157)$ \\
Baseline $\mathrm{SpO}_{2}$ (\%) & $-0.44 \pm 0.22$ & $-0.45 \pm 0.22$ \\
& $(0.001)$ & $(0.001)$ \\
Cardiovascular disease & $4.47 \pm 2.71$ & $5.27 \pm 2.38$ \\
& $(0.064)$ & $(0.019)$
\end{tabular}

Association between breath-holding measurements at admission and the adverse composite primary outcome in COVID-19 (multivariable logistic regression). Data shown are $\beta \pm \operatorname{SEM}$ ( $p$ value); $\beta$ describes the increase in log-odds of the adverse outcome per change in exposure variable. Primary model: The breath-holding measurements significantly improved the model (likelihood ratio $0.0073, p=0.02$ ) over a reference model with covariates only (body mass index, baseline $\mathrm{SpO}_{2}$, cardiovascular disease [1 = Present, $0=$ Absent], plus age and sex [not shown]). $p$ values are based on likelihood ratio tests. The parsimonious model is a simplified and refined version of the primary model (age, sex, and ventilatory response were removed $[p>0.2]$; Intercept $=25.31 \pm 17.31$ ). The potential predictive value of the model is illustrated in Fig. 2D and a tool for risk calculation is provided in Additional file 2

feature of COVID-19 does not support the notion that vigorous ventilatory drive promotes self-inflicted lung injury (P-SILI) [31-33], but rather is consistent with "silent hypoxemia" as a prevalent characteristic in COVID-19 [13-15, 34, 35]. A third breath-holding variable, the ventilatory response to 20-s breath holds, was not associated with the primary outcome. Each analysis demonstrated that associations between breath-holding variables and the adverse outcomes (as well as outcome prediction) were seen above and beyond baseline saturation (and other commonly-available clinical measures). Overall, the current study provides unique insight into the differential physiological characteristics of patients who exhibit adverse outcomes $v$. those who do not. We consider that this knowledge has the potential to be used in future tools to identify patients at elevated risk of adverse outcomes in COVID-19.

\section{Novel physiological insights Desaturation}

Early signs of gas exchange deficits in patients who later develop severe COVID-19-such as regional ventilation/perfusion (V/Q) heterogeneity and reduced lung gas volumes-have been inferred from chest imaging
$[5,14,15]$; however, functional evidence of this notion is limited. Here, we show that breath-holding unmasks a greater COVID-19-related decline in gas exchange in those who progress to severe disease versus those who do not. Specifically, after adjusting for baseline $\mathrm{SpO}_{2}$, breath-holding desaturation is an independent risk factor for adverse outcomes in COVID-19. Indeed, breathholding is expected to provide unique information on gas exchange deficits on the basis that V/Q heterogeneity (lower $\mathrm{V} / \mathrm{Q}$ regions readily desaturate during apnea) [16] and reduced lung gas volumes (greater decline in alveolar $\mathrm{PO}_{2}$ per unit time) $[17,18]$ influence desaturation speed independently of baseline $\mathrm{SpO}_{2}$. Of note, baseline $\mathrm{SpO}_{2}$ can be insensitive to reduced $\mathrm{PaO}_{2}$ when on the plateau of the $\mathrm{SpO}_{2} / \mathrm{PaO}_{2}$ curve. Our findings also withstood adjustment for obesity and pre-existing cardiovascular disease as confounders, suggesting that these particular non-COVID sources of variability do not explain away the associations observed.

\section{Ventilatory control}

The finding that longer maximal breath-hold duration $\left(\mathrm{SpO}_{2}\right.$-adjusted [30]) confers risk in COVID-19 suggests that blunted ventilatory control responses [26-28] may precede severe versus milder disease. Supporting this notion, absence of dyspnea (Borg $=0$ ) was also an independent risk factor for adverse outcomes in the current study (n.b. impaired chemosensitivity may mitigate dyspnea [36-40]). Notably, COVID-19 is associated with anosmia/dysgeusia, and investigators have previously speculated infection at the carotid bodies [21] or centrally [34]. Regardless, it is unclear whether ventilatory control effects of COVID-19 are causally involved in respiratory failure or simply a marker of more severe viral illness. Indeed, interventional studies using ventilatory stimulants could shed light on the putative causal pathways. We also observed that patients who did not develop severe disease had shorter breath-hold durations $v$. healthy controls; thus, such individuals may escape adverse outcomes of COVID-19 partly through a more robust ventilatory control defense against hypoxia/hypercapnia. Overall, our data do not support the concept that robust chemoreflexes exacerbate lung injury via greater chemoreflex-related transpulmonary pressures (P-SILI) [23, 24]. Instead, our study suggests that blunted ventilatory control in the face of hypoxemia (i.e. "silent hypoxemia") is additionally deleterious in COVID-19 [19, 20, 22, 34, 35]. Nonetheless, our findings suggest that breath-holding duration may be a clinically important biomarker for identifying risk of subsequent respiratory failure regardless of the underlying mechanisms. 


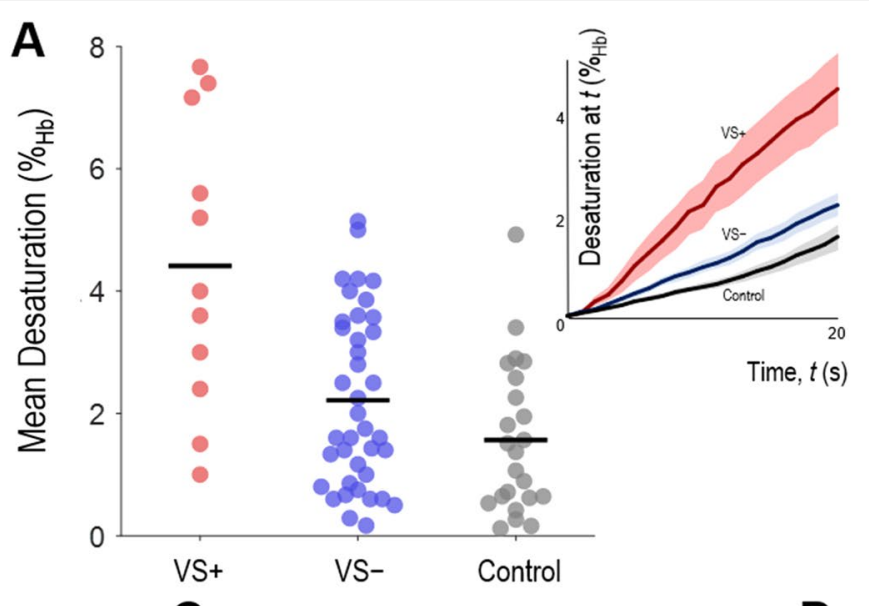

C

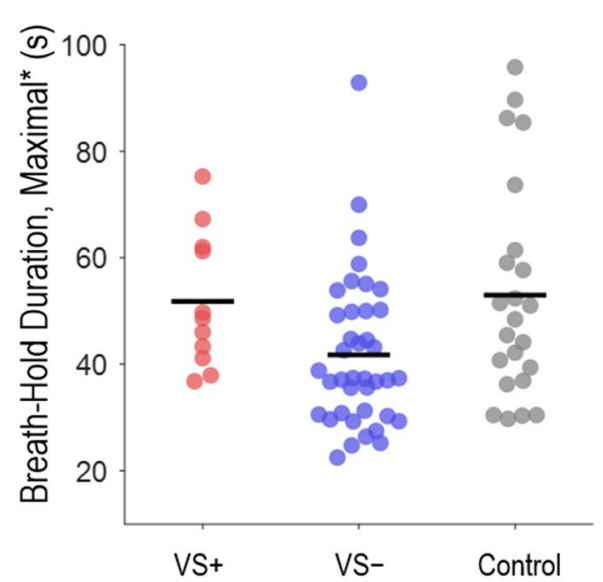

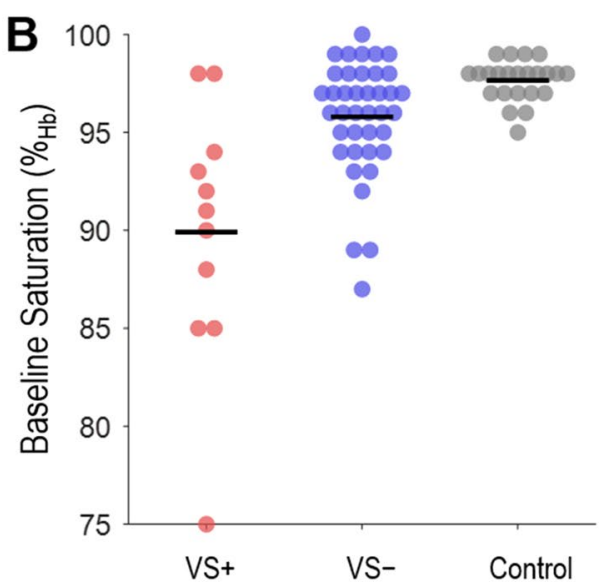

D

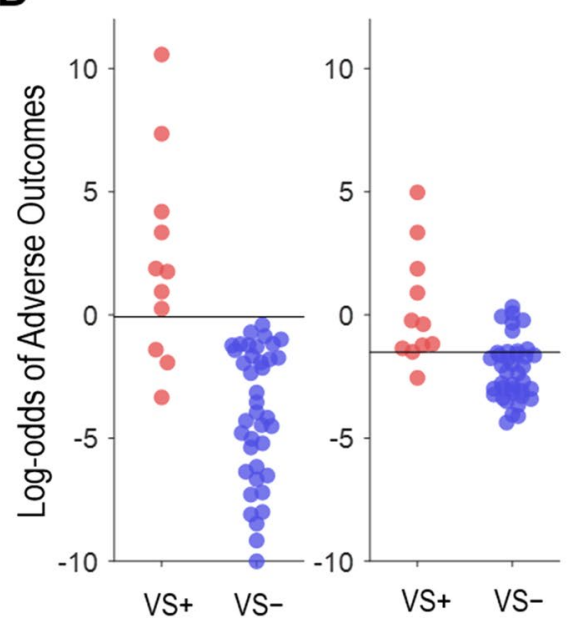

Fig. 2 Individual breath-holding measurements in COVID-19 patients who experienced the adverse primary composite outcome (and required ventilatory support "VS +", N=11), in COVID-19 patients without adverse outcomes ("VS -", N=39), and controls ( $N=23$ ). Horizontal black bars overlying individual data indicate group mean values. A. Mean desaturation after 20-s, unadjusted. Inset: desaturation profile for each group (mean \pm SEM desaturation at any time, delay corrected). B. Baseline oxygen saturation, a potential confounder for mean desaturation, was different between groups and independently associated with adverse COVID-19 outcomes. C. Maximal breath-hold duration ( ${ }^{*}$ shown adjusted for baseline $\mathrm{SpO}_{2}\left[+2.6 \mathrm{~s} \mathrm{per} \mathrm{\%} \mathrm{Hb}_{\text {b }}\right.$ below 97.6] and mean desaturation [+ $0.6 \mathrm{~s}$ per \% $\%_{\mathrm{Hb}}$ above 1.57]); shorter adjusted durations were interpreted as increased chemosensitivity (or sensitivity to dyspnea). Data on ventilatory response to breath-holds (not shown) were similar between groups. D. Left: Multivariable logistic regression model output for each individual patient (parsimonious model, Table 2) shows that mean desaturation and maximal breath-hold duration (+ covariates baseline $\mathrm{SpO}_{2}$, body mass index, cardiovascular disease) provides good outcome discrimination (threshold $\sim 0$ ); a score of 0 represents $50 \%$ probability of the adverse primary outcome. Each 1-point increase represents a log (2.7-fold) increase in likelihood of the primary outcome. Right: Reference model without breath-holding measures (baseline $\mathrm{SpO}_{2}$, body mass index, cardiovascular disease) showed significantly reduced outcome discrimination ( $p=0.007$, random permutation analysis)

\section{Clinical implications}

Understanding risk factors for adverse outcomes of COVID-19 has been the focus of intense research over the last 12 months. To date, notable studies examining risk factors have been retrospective in design [2, 4-9, 14]. In the current prospective study, greater breath-holding desaturation and reduced maximal breath-holding duration were associated with adverse outcomes in COVID19 independently of baseline $\mathrm{SpO}_{2}$, and early analysis suggests that the approach has potential predictive value.
Exploratory inclusion of existing biomarkers C-reactive problem and d-dimer, and adjustments for haemoglobin levels did not change our findings. Our translational work therefore demonstrates the feasibility of using physiological testing to estimate the risk of adverse COVID-19 outcomes days in advance of patient deterioration, enabling prioritization of limited resources to the high risk patients who need them most (Fig. 2D), and providing a window for early administration of medical therapies (e.g. dexamethasone) prior to advanced disease progression, 
especially in pandemic epicenters (an excel prognostic tool is also provided for academic evaluation). These findings are consistent with anecdotal reports of clinicians in Italy and beyond successfully using exertional test-derived altered $\mathrm{SpO}_{2}$ values to triage patients with Covid-19 and hospitalization those with post-exertional greater desaturation [10-12]. However, in contrast to exertional tests (i.e. 6-min walking test), breath-holding does not require increased energy expenditure or cardiac output, and obviates walking and associated bystander/ caregiver contamination. Measurement of these simple, novel surrogates capturing pulmonary and chemoreflex risk factors requires minimal inventory (i.e. a means to record oximetry and a timing device) and could feasibly provide a useful means to estimate risks of future deterioration in under-resourced circumstances, should future validation studies support this concept.

\section{Methodological considerations}

This study has several limitations. First, while the sample size provided sufficient power to confirm our primary hypothesis (see Additional file 1: Statistical Analysis), we did not have an additional sample for an independent validation analysis (study is forthcoming). However, random permutation analysis and leave-one-out cross validation provided rigor and reassurance that results were not the trivial consequence of overfitting. Second, the number of covariates (potential confounders) included in our model analyses may raise concerns, yet we emphasize that simulations demonstrated that statistical power was not meaningfully reduced by the inclusion of uncorrelated covariates and that the inclusion or removal of covariates did not strength or weaken the associations. Third, given the narrow race/ethnicity of our study population, we cannot generalize our findings to all potential patient populations globally. In addition, our analysis suggests that breath-holding variables are associated with adverse outcomes independent of sex, but we are unable to conclude whether breath-holding variables are associated with adverse outcomes specifically within men or within women, or if sex-specific models might be needed. A larger study of women and men is needed to address these questions. Fourth, breath-holding required cooperation so patients who necessitated immediate ventilatory support were not studied. Nevertheless, we highlight that people requiring urgent intervention are, by definition, readily triaged and beyond the scope of this work. Fifth, the maximal breath-hold variable, but not the ventilatory response variable, suggested increased chemosensitivity was protective. We emphasize, however, that measurement of the ventilatory response was more complex (requires assumptions of nasal breathing and effective linearization of nasal pressure). Of note, the ventilatory response variable is susceptible to measurement noise in the absence of a sealed oronasal mask and pneumotachometer [26] and thus potentially unreliable. Despite these limitations, we believe our findings are clinically important and deserve further study.

\section{Conclusions}

Breath-holding measurements of susceptibility to rapid desaturation and ventilatory control sensitivity are associated with progression to respiratory failure in COVID19: greater desaturation during breath-holds (interpreted as greater gas exchange deficit) and longer maximal breath-holds (interpreted as lower chemosensitivity) are independent risk factors. Simplified physiological measures of gas exchange and neurophysiological deficits in COVID-19 may hold utility for future translational use in early triage to scarce health care resources or early administration of medical interventions. Our study also raises the possibility that blunted ventilatory control is a therapeutic target for preventing severe disease in COVID-19, a concept that will require interventional studies to assess.

\section{Supplementary Information}

The online version contains supplementary material available at https://doi. org/10.1186/s13054-021-03630-5.

Additional file 1: Supplemental text (methods and results) and tables.

Additional file 2: Tool to assess probability of the primary adverse outcome (for academic evaluation).

Acknowledgements

We are sincerely thankful to all the Italian sleep technicians that worked on this project, especially Valeria Di Stefano, Francesca Gregorini and Anna Motta, for their essential contribution to data collection.

Authors' contributions

Study design: LM, SS. Data collection: EP, LC, SJ, FF, LP, CL, GV. Data analysis: LM, SS. Interpretation of results: All authors. Drafting of the manuscript: LM, AM, SS Editing of the manuscript for important intellectual content: All authors.

\section{Funding}

This study was not directly funded. LM is supported by Flinders University. SJ is supported by an NHMRC Early Career Fellowship (1139745). FF is supported by the "Ricerca Corrente" funding of ICS Maugeri Spa SB. AM is supported by $\mathrm{NIH}$. SS is supported by the NIH NHLBI (R01HL146697).

\section{Availability of data and materials}

All the individual participant and summary data collected during the trial will be shared upon request, after de-identification. Additionally, study protocol, statistical analysis plan and informed consent will be made available. Data will be available immediately following publication and ending 5 years following article publication with researchers who provide a methodologically sound proposal to achieve aims in such approved proposal. Proposals should be directed to LM ludovico.messineo@yahoo.it or EP e.perger@auxologico.it; access will require a data use agreement. 


\section{Declarations}

\section{Ethics approval}

This study was approved by the Italian Auxologico Institute Ethics Committee (number: 2020_04_03_03) on April 3rd, 2020, which was the reference center for recruitment of COVID-19 patients, and by the Monash Health HREC (number: RES-20-0000-264A) on April 29th, 2020, which was the main reference for recruitment of healthy individuals. The study was also approved by the Brescia local Ethics Committee (number: 4068) on April 17th, 2020 and Pavia local Ethics Committee (number: 2412) on April 20th, 2020. All participants provided verbal or written informed consent upon enrolment (the study was exempted from written consent in some centers to limit the spread of the infection). The study conformed to the standards set by the latest revision of the Declaration of Helsinki. This study also adhered to both STROBE (Strengthening the Reporting of Observational Studies in Epidemiology) and TRIPOD (Transparent Reporting of a multivariable prediction model for Individual Prognosis or Diagnosis) reporting guidelines.

\section{Consent for publication}

NA.

\section{Competing interests}

AW works as a consultant for Apnimed, Nox, Inspire, and Somnifix. He has received grants from Sanofi and Somnifix. He also has a financial interest in Apnimed Corp., a company developing pharmacologic therapies for sleep apnea. AW interests were reviewed and are managed by Brigham and Women's Hospital and Partners HealthCare in accordance with their conflict of interest policies. SS reports grants and personal fees from Apnimed, personal fees from Nox Medical, personal fees from Merck, personal fees from Inspire, grants from Prosomnus, grants from Dynaflex, outside the submitted work. In addition, SS has a patent pending related to wearable oximetry technology for sleep apnea diagnosis/phenotyping, unrelated to breath-holding. GH reports non-financial support from Resmed, non-financial support from Philips Respironics, non-financial support from Air Liquide Healthcare, outside the submitted work. AM reports income related to medical education from Merck and Livanova; ResMed provided a philanthropic donation to UC San Diego. $L M, E P, L C S J, F F, L P, P T, C L, G V$, GP have no conflicts of interest to disclose.

\section{Author details}

'Division of Sleep and Circadian Disorders, Departments of Medicine and Neurology, Brigham \& Women's Hospital \& Harvard Medical School, Boston, MA, USA. ${ }^{2}$ Adelaide Institute for Sleep Health (AISH), Flinders Health and Medical Research Institute (FHMRI), Flinders University, 5 Laffer Drive, Bedford Park, Adelaide, SA 5043, Australia. ${ }^{3}$ Istituto Auxologico Italiano IRCSS, Sleep Medicine Center, Department of Cardiology, San Luca Hospital, Milano, Italy. ${ }^{4}$ Department of Medicine and Surgery, University of Milano-Bicocca, Milan, Italy. ${ }^{5}$ Respiratory Medicine and Sleep Laboratory, Department of Experimental and Clinical Sciences, University of Brescia and Spedali Civili, Brescia, Italy. ${ }^{6}$ Department of Internal Medicine, Spedali Civili, Brescia, Italy. ${ }^{7}$ Monash Lung and Sleep, Monash Medical Centre, Clayton, VIC, Australia. ${ }^{8}$ School of Clinical Sciences, Monash University, Melbourne, VIC, Australia. ${ }^{9}$ Monash Partners Epworth, Victoria, Australia. ${ }^{0}$ Maugeri Institute IRCCS, Sleep Medicine Center, Pavia, Italy. ${ }^{11}$ School of Information Technology and Electrical Engineering, The University of Queensland, Brisbane, Australia. ${ }^{12}$ University of California San Diego, La Jolla, CA, USA. ${ }^{13}$ Department of Allergy Immunology and Respiratory Medicine and Central Clinical School, The Alfred and Monash University, Melbourne, Australia.

\section{Received: 23 April 2021 Accepted: 6 June 2021}

Published online: 14 June 2021

\section{References}

1. Rosenbaum L. Facing Covid-19 in Italy - Ethics, Logistics, and Therapeutics on the Epidemic's Front Line. N Engl J Med. 2020.

2. Zhou F, Yu T, Du R, Fan G, Liu Y, Liu Z, et al. Clinical course and risk factors for mortality of adult inpatients with COVID-19 in Wuhan, China: a retrospective cohort study. Lancet. 2020;395(10229):1054-62.
3. WHO. Clinical management of severe acute respiratory infection when novel coronavirus ( $\mathrm{nCoV}$ ) infection is suspected; interim guidance, May 27th. 2020 [cited 2020 July, 7th]; https://apps.who.int/iris/bitstream/ handle/10665/332196/WHO-2019-nCoV-clinical-2020.5-eng.pdf?seque nce $=1$ \&isAllowed $=y$.

4. Bello-Chavolla OY, Bahena-Lopez JP, Antonio-Villa NE, Vargas-Vazquez A, Gonzalez-Diaz A, Marquez-Salinas A et al. Predicting mortality due to SARS-CoV-2: a mechanistic score relating obesity and diabetes to COVID-19 outcomes in Mexico. J Clin Endocrin Metab. 2020;105(8).

5. Angeli E, Dalto S, Marchese S, Setti L, Bonacina M, Galli F, et al. Prognostic value of CT integrated with clinical and laboratory data during the first peak of the COVID-19 pandemic in Northern Italy: a nomogram to predict unfavorable outcome. Eur J Radiol. 2021;137:109612.

6. Ruan Q, Yang K, Wang W, Jiang L, Song J. Clinical predictors of mortality due to COVID-19 based on an analysis of data of 150 patients from Wuhan. China Intensive Care Med. 2020;46(5):846-8.

7. Xie J, Hungerford D, Chen H, Abrams ST, Li S, Wang G, et al. Development and external validation of a prognostic multivariable model on admission for hospitalized patients with COVID-19. medRxiv. 2020.

8. Shi Y, Yu X, Zhao H, Wang H, Zhao R, Sheng J. Host susceptibility to severe COVID-19 and establishment of a host risk score: findings of 487 cases outside Wuhan. Crit Care. 2020;24(1):108.

9. Sun H, Jain A, Leone MJ, Alabsi HS, Brenner LN, Ye E, et al. COVID-19 outpatient screening: a prediction score for adverse events. medRxiv. 2020.

10. Paglia S, Storti E, Magnacavallo A, Cianci V, Cibinel GA, Fabbri A, et al. Rapporto Prima Linea Covid-19 assetto organizzativo gestionale dei PS/DEA nell'ambito di focolaio epidemico o pre-epidemico. 2020 [cited 2020 July, 14th]; http://www.quotidianosanita.it/allegati/alleg ato3277121.pdf.

11. Bertolini G, Nattino G. Pazienti COVID-19: la cura inizia a casa. Un progetto di monitoraggio attivo a Casale Monferrato e Torino 2020 [cited 2020 July, 7th]; http://www.fondazionebuzziunicem.org/documents/459904/ 466757/COVID-lacurainziaacasa.pdf/66404644-6828-110a-8129-cd9ce fadf581.

12. Greenhalgh $T$, Javid B, Knight M, Inada-Kim M. What is the efficacy and safety of rapid exercise tests for exertional desaturation in Covid-19? : Oxford Covid-19 evidence service; 2020; https://www.researchgate.net/ publication/340934179_What_is_the_efficacy_and_safety_of_rapid_ exercise_tests_for_exertional_desaturation_in_covid-19.

13. Goodacre S, Thomas B, Lee E, Sutton L, Loban A, Waterhouse S et al. Postexertion oxygen saturation as a prognostic factor for adverse outcome in patients attending the emergency department with suspected COVID19: a substudy of the PRIEST observational cohort study. Emerg Med J. 2020.

14. Wang S, Zha Y, Li W, Wu Q, Li X, Niu M, et al. A fully automatic deep learning system for COVID-19 diagnostic and prognostic analysis. Eur Respir J. 2020;56(2).

15. Bernheim A, Mei X, Huang M, Yang Y, Fayad ZA, Zhang N, et al. Chest CT findings in coronavirus disease-19 (COVID-19): relationship to duration of infection. Radiology. 2020;295(3):200463.

16. Sands SA, Edwards BA, Kelly VJ, Davidson MR, Wilkinson MH, Berger PJ. A model investigation of the impact of ventilation-perfusion mismatch on oxygenation during apnea in preterm infants. J Theor Biol. 2010. https:// doi.org/10.1016/j.jtbi.2010.03.041.

17. Sands SA, Edwards BA, Kelly VJ, Davidson MR, Wilkinson MH, Berger PJ. A model analysis of arterial oxygen desaturation during apnea in preterm infants. PLoS Comput Biol. 2009;5(12):e1000588.

18. Findley LJ, Ries AL, Tisi GM, Wagner PD. Hypoxemia during apnea in normal subjects: mechanisms and impact of lung volume. J Appl Physiol. 1983;55(6):1777-83.

19. Couzin-Frankel J. The mystery of the pandemic's "happy hypoxia." Science. 2020;368(6490):455-6.

20. Widysanto A, Wahyuni TD, Simanjuntak LH, Sunarso S, Siahaan SS, Haryanto $\mathrm{H}$, et al. Happy hypoxia in critical COVID-19 patient: a case report in Tangerang, Indonesia. Physiol Rep. 2020;8(20):e14619.

21. Villadiego J, Ramírez-Lorca R, Cala F, Labandeira-García JL, Esteban M, Toledo-Aral JJ, et al. Is Carotid body infection responsible for silent hypoxemia in COVID-19 patients? Function. 2020;2(1).

22. Tobin MJ, Laghi F, Jubran A. Why COVID-19 silent hypoxemia is baffling to physicians. Am J Respir Crit Care Med. 2020 
23. Gattinoni L, Marini JJ, Camporota L, et al. Reply to Tobin, Respiratory drive measurements do not signify conjectural patient self-inflicted lung injury. Am J Respir Crit Care Med. 2021;203(1):143-4.

24. Marini JJ, Gattinoni L. Management of COVID-19 respiratory distress. JAMA. 2020;323(22):2329-30.

25. Tobin MJ, Jubran A, Laghi F. Respiratory drive measurements do not signify conjectural patient self-inflicted lung injury. Am J Respir Crit Care Med. 2021;203(1):142-3.

26. Messineo L, Taranto-Montemurro L, Azarbarzin A, Oliveira Marques MD, Calianese N, White DP, et al. Breath-holding as a means to estimate the loop gain contribution to obstructive sleep apnoea. J Physiol. 2018;596(17):4043-56

27. Stanley NN, Cunningham EL, Altose MD, Kelsen SG, Levinson RS, Cherniack NS. Evaluation of breath holding in hypercapnia as a simple clinical test of respiratory chemosensitivity. Thorax. 1975;30(3):337-43.

28. Trembach N, Zabolotskikh I. Breath-holding test in evaluation of peripheral chemoreflex sensitivity in healthy subjects. Respir Physiol Neurobiol. 2017;235:79-82.

29. Duca A, Piva S, Foca E, Latronico N, Rizzi M. Calculated Decisions: BresciaCOVID respiratory severity scale (BCRSS)/algorithm. Emerg Med Pract. 2020;22(5 Suppl):CD1-2.

30. Bruce CD, Vanden Berg ER, Pfoh JR, Steinback CD, Day TA. Prior oxygenation, but not chemoreflex responsiveness, determines breath-hold duration during voluntary apnea. Physiol Rep. 2021;9(1):e14664.

31. Marini JJ, Gattinoni L. Management of COVID-19 respiratory distress. JAMA. 2020.

32. Mascheroni D, Kolobow T, Fumagalli R, Moretti MP, Chen V, Buckhold D. Acute respiratory failure following pharmacologically induced hyperventilation: an experimental animal study. Intensive Care Med. 1988;15(1):8-14.

33. Esnault P, Cardinale M, Hraiech S, Goutorbe P, Baumstrack K, Prud'homme $\mathrm{E}$, et al. High respiratory drive and excessive respiratory efforts predict relapse of respiratory failure in critically III patients with COVID-19. Am J Respir Crit Care Med. 2020.

34. Swenson KE, Ruoss SJ, Swenson ER. The pathophysiology and dangers of silent hypoxemia in COVID-19 lung injury. Ann Am Thoracic Soc. 2021.

35. Simonson TS, Baker TL, Banzett RB, Bishop T, Dempsey JA, Feldman JL, et al. Silent hypoxaemia in COVID-19 patients. J Physiol. 2021;599(4):1057-65.

36. Davidson JT, Whipp BJ, Wasserman K, Koyal SN, Lugliani R. Role of the carotid bodies in breath-holding. N Engl J Med. 1974;290(15):819-22.

37. Paton JY, Swaminathan S, Sargent CW, Keens TG. Hypoxic and hypercapnic ventilatory responses in awake children with congenital central hypoventilation syndrome. Am Rev Respir Dis. 1989;140(2):368-72.

38. Gigliotti F. Mechanisms of dyspnea in healthy subjects. Multidiscip Respir Med. 2010;5(3):195-201.

39. Kikuchi Y, Okabe S, Tamura G, Hida W, Homma M, Shirato K, et al. Chemosensitivity and perception of dyspnea in patients with a history of near-fatal asthma. N Engl J Med. 1994;330(19):1329-34.

40. Molfino NA, Nannini LJ, Martelli AN, Slutsky AS. Respiratory arrest in nearfatal asthma. N Engl J Med. 1991;324(5):285-8.

\section{Publisher's Note}

Springer Nature remains neutral with regard to jurisdictional claims in published maps and institutional affiliations.
Ready to submit your research? Choose BMC and benefit from:

- fast, convenient online submission

- thorough peer review by experienced researchers in your field

- rapid publication on acceptance

- support for research data, including large and complex data types

- gold Open Access which fosters wider collaboration and increased citations

- maximum visibility for your research: over 100M website views per year

At BMC, research is always in progress.

Learn more biomedcentral.com/submissions 